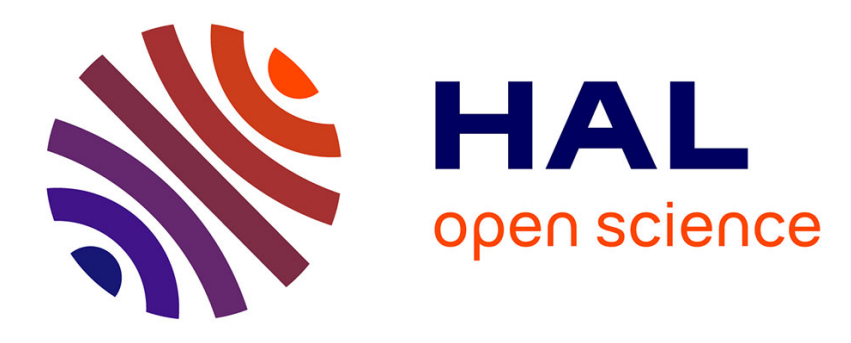

\title{
Modeling of Branched Needle Microstructures at the Edge of a Martensite Laminate
}

\author{
R. James, R. Kohn, T. Shield
}

\section{To cite this version:}

R. James, R. Kohn, T. Shield. Modeling of Branched Needle Microstructures at the Edge of a Martensite Laminate. Journal de Physique IV Proceedings, 1995, 05 (C8), pp.C8-253-C8-259. 10.1051/jp4:1995835 . jpa-00254084

\section{HAL Id: jpa-00254084 https://hal.science/jpa-00254084}

Submitted on 1 Jan 1995

HAL is a multi-disciplinary open access archive for the deposit and dissemination of scientific research documents, whether they are published or not. The documents may come from teaching and research institutions in France or abroad, or from public or private research centers.
L'archive ouverte pluridisciplinaire HAL, est destinée au dépôt et à la diffusion de documents scientifiques de niveau recherche, publiés ou non, émanant des établissements d'enseignement et de recherche français ou étrangers, des laboratoires publics ou privés. 


\title{
Modeling of Branched Needle Microstructures at the Edge of a Martensite Laminate
}

\author{
R.D. James, R.V. Kohn* and T.W. Shield \\ Department of Aerospace Engineering and Mechanics, 107 Akerman Hall, 110 Union Street S.E., \\ University of Minnesota, Minneapolis, MN 55455, U.S.A. \\ * Courant Institute of Mathematical Sciences, 251 Mercer Street, New York, NY 10012, U.S.A.
}

\begin{abstract}
We present models of the microstructure of martensite that occurs when twinned layers of martensite meet a pure variant. This microstructure often occurs below the $A_{s}$ temperature, with loading that favors the passage from one pure variant of martensite to another. This microstructure gives a mechanism for variant rearrangement. In this microstructure, the layers are observed to pinch down to sharply pointed needles. If the laminate is sufficiently long, branching of the needles is observed. In materials with a reasonably large transformation strain, the needles exhibit a characteristic bent shape; the bending is in the opposite sense as might be expected from the overall shear. Our analytical and numerical results imply that this microstructure forms as a result of energy minimization in the transition layer between the martensite laminate and the pure variant. The bending of the needles plays a key role in lowering the energy of this transition layer.
\end{abstract}

\section{GEOMETRICALLY LINEAR AND NONLINEAR THEORIES OF MARTENSITE}

A picture of the microstructure of interest here is shown in Figure 1, taken from the biaxial loading experiments of [7].

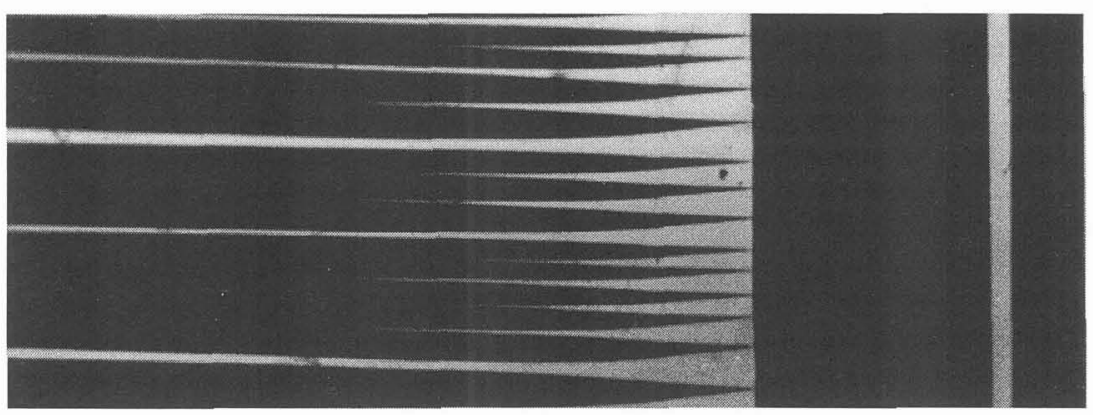

Figure 1. Branched needle microstructure.

When a twinned laminate of martensite meets a pure variant as in Figure 1, there are certain matching conditions that must hold which are exactly analogous to those of the crystallographic theory of martensite. We prefer to think of them as a consequence of energy minimization (see [3], [4] and [5] for the explanation of how the crystallographic theory follows from energy minimization). In this viewpoint the bulk free energy density of the specimen is a function $\varphi$ of the deformation gradient, $\mathbf{F}=\nabla \mathbf{y}$, (a $3 \times 3$ matrix) and the temperature $\theta$. Throughout this paper we hold $\theta$ fixed below $A_{s}$ and so we omit it from the notation. The deformation $\mathbf{y}(\mathbf{x})$ maps a suitable reference configuration into 3-dimensional space. The total bulk free energy is given by the integral

$$
\int_{\Omega} \varphi(\nabla \mathbf{y}(\mathbf{x})) d \mathbf{x} .
$$

A key feature of $\varphi$ is that it has energy wells (minima), one at the deformation gradient of each variant. For the purposes of this discussion we can focus on just two of those energy wells corresponding to the two variants of martensite in question. A second key feature, arising from basic principles, is that $\varphi$ is frame-indifferent (i.e., unaffected by overall rotations), which means that $\varphi(\mathbf{R F})=\varphi(\mathbf{F})$. Here, $S O(3)$ denotes the set of all rotation matrices, $S O(3)=\left\{\mathbf{R}: \mathbf{R}\right.$ is a $3 \times 3$ matrix satisfying $\mathbf{R}^{T} \mathbf{R}=\mathbf{I}$, $\left.\operatorname{det} \mathbf{R}=+1\right\}$, a superscript $T$ denotes the transpose and $\mathbf{I}$ is the identity matrix. Frame-indifference implies that if a $3 \times 3$ matrix $\mathbf{F}$ minimizes $\varphi$, then so does $\mathbf{R F}$ where $\mathbf{R}$ belongs to $S O(3)$; for simplicity we call this set $S O(3) \mathbf{F}$. In [4] 
it is shown that any two energy wells (arising from any cubic-to-orthorhombic, tetragonal-to-monoclinic or similar transformation) can be put in the canonical form $S O(3) \mathbf{A}, S O(3) \mathbf{B}$ by a linear change of variables $\mathbf{x} \rightarrow \mathbf{G x}$, where $\mathbf{A}$ and $\mathbf{B}$ are the simple shear matrices

$$
\mathbf{A}=\left(\begin{array}{lll}
1 & \delta & 0 \\
0 & 1 & 0 \\
0 & 0 & 1
\end{array}\right), \mathbf{B}=\left(\begin{array}{ccc}
1 & -\delta & 0 \\
0 & 1 & 0 \\
0 & 0 & 1
\end{array}\right)
$$

We assume $\varphi \geq 0$ and $\varphi=0$ exactly on the set $S O(3) \mathbf{A} \cup S O(3) \mathbf{B}$ with $\mathbf{A}$ and $\mathbf{B}$ given by (2).

In terms of bulk energy minimization, the overall calculation referred to at the beginning of this section is

$$
\mathbf{R B}-(\lambda \mathbf{A}+(1-\lambda) \mathbf{B})=\mathbf{b} \otimes \mathbf{m} .
$$

This is to be solved for the rotation matrix $\mathbf{R}$, and the volume fraction $\lambda$, and the vectors $\mathbf{b}$ and $\mathbf{m}$. Here, $\mathbf{b} \otimes \mathbf{m}$ is the $3 \times 3$ matrix with components $b_{i} m_{j}$ in a Cartesian coordinate system. The condition (3) is sufficient to ensure that the bulk energy in the transition layer between the laminate and the pure variant (see Figure 2) can be made arbitrarily small by refining the layers. By scaling, (3) is also necessary and sufficient for the deformation defined on the transition layer to be periodic along the layer. It can easily be shown that for each $\lambda$ in the interval $[0,1]$ there is a unique solution $(\widehat{\mathbf{R}}, \widehat{\mathbf{b}} \otimes \widehat{\mathbf{m}})$ of (3), which takes the form:

$$
\widehat{\mathbf{R}}=\left(\begin{array}{ccc}
\frac{1-\lambda^{2} \delta^{2}}{1+\lambda^{2} \delta^{2}} & \frac{2 \lambda \delta}{1+\lambda^{2} \delta^{2}} & 0 \\
\frac{-2 \lambda \delta}{1+\lambda^{2} \delta^{2}} & \frac{1-\lambda^{2} \delta^{2}}{1+\lambda^{2} \delta^{2}} & 0 \\
0 & 0 & 1
\end{array}\right), \widehat{\mathbf{b}} \otimes \widehat{\mathbf{m}}=\frac{-2 \lambda \delta}{1+\lambda^{2} \delta^{2}}(\lambda \delta, 1,0) \otimes(1, \delta(\lambda-1), 0) .
$$

Thus, unlike in the crystallographic theory of martensite, the condition (3) does not determine the volume fraction. Thus by changing $\lambda$ from 0 to 1 the microstructure can pass on a low energy path from pure variant $\mathbf{A}$ to pure variant $\mathbf{B}$. These laminates are typically observed [2] patched together to form more complicated energy minimizing microstructures. The normal $\widehat{\mathrm{m}}$ comes out to be nearly, but not precisely, parallel to the reciprocal twin boundary normal, as can be seen in Figure 1. We view the equations (3) and (4) as providing the boundary conditions for a more refined model of the transition layer.

In geometrically linear theory (see [6], [9], [10] and [12]) the condition of frame-indifference is replaced by its linearized counterpart, which means that the corresponding geometrically linear energy $\varphi_{\operatorname{lin}}(\mathbf{H})$ as a function of the displacement gradient $\mathbf{H} \approx \nabla(\mathbf{y}(\mathbf{x})-\mathbf{x})$ satisfies $\varphi_{\text {lin }}(\mathbf{H}+\mathbf{W})=\varphi_{\text {lin }}(\mathbf{H})$ for all skew matrices $\mathbf{W}=-\mathbf{W}^{T}$. The wells in geometrically linear theory become $\left(\frac{1}{2}\left(\mathbf{A}+\mathbf{A}^{T}\right)-\mathbf{I}\right)+\mathbf{W}$, $\left(\frac{1}{2}\left(\mathbf{B}+\mathbf{B}^{T}\right)-\mathbf{I}\right)+\mathbf{W}$, where $\mathbf{W}$ is any skew matrix. Letting $\mathbf{E}_{A}=\frac{1}{2}\left(\mathbf{A}+\mathbf{A}^{T}\right)-\mathbf{I}, \mathbf{E}_{B}=\frac{1}{2}\left(\mathbf{B}+\mathbf{B}^{T}\right)-\mathbf{I}$, the analog of (2) in geometrically linear theory is

$$
\mathbf{E}_{B}+\mathbf{W}-\left(\lambda \mathbf{E}_{A}+(1-\lambda) \mathbf{E}_{B}\right)=\mathbf{b} \otimes \mathbf{m} .
$$

Equation (5) has the solution (which should be viewed as an approximation to the true solution (4) in the limit of small strains and rotations):

$$
\mathbf{W}=\left(\begin{array}{ccc}
0 & \delta \lambda & 0 \\
-\delta \lambda & 0 & 0 \\
0 & 0 & 1
\end{array}\right), \quad \mathbf{b} \otimes \mathbf{m}=-2 \delta \lambda(010) \otimes(100)
$$

\section{MODEL OF THE TRANSITION LAYER - GEOMETRICALLY NONLINEAR THEORY}

To explain in simple terms the origins of our models and the reasons for needle bending, consider the boundary value problem associated with Figure 2 . Here we seek an energy minimizing deformation defined on the gray region consistent with the boundary conditions shown there, which are obtained from the overall solution (4). 


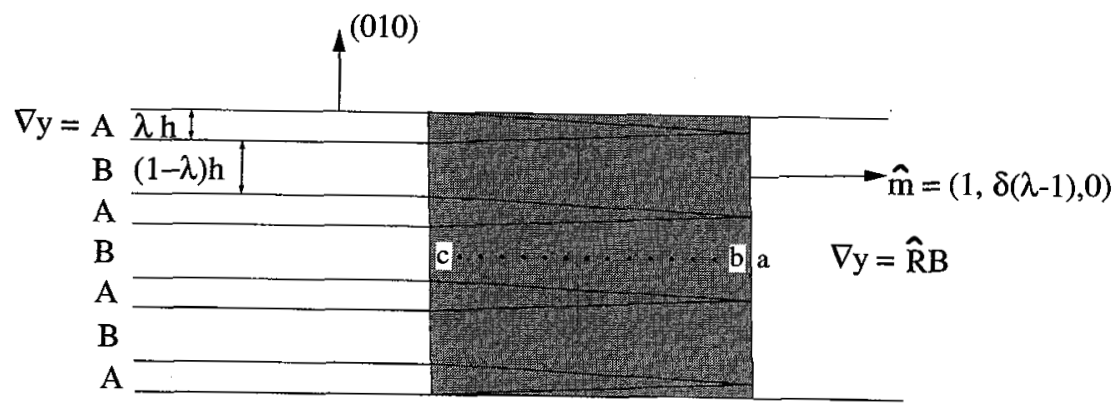

Figure 2. Boundary value problem for the transition layer (shaded region).

The deformation gradient at point $a$ is not expected to be precisely $\widehat{\mathbf{R} B}$, because of relaxation near the interface (see Section 4), but let us neglect this momentarily. To begin the solution in the gray region, we seek a deformation gradient matrix $\widetilde{\mathbf{A}}$ defined at point $b$ in Figure 2 which differs from $\widehat{\mathbf{R}} \mathbf{B}$ by a rank-1 matrix of the form $\mathbf{c} \otimes \widehat{\mathbf{m}}$ (so the resulting deformation is compatible). To have low energy, we would hope to find such a matrix $\widetilde{\mathbf{A}}$ near the energy well $S O(3) \mathbf{A}$. This suggests that we study the problem $\widetilde{\mathbf{A}}-\widehat{\mathbf{R}} \mathbf{B}=\mathbf{c} \otimes \widehat{\mathbf{m}}$, $\widetilde{\mathbf{A}} \approx \widetilde{\mathbf{R}} \mathbf{A}, \widetilde{\mathbf{R}} \in S O(3)$. Indeed, because $\widehat{\mathbf{m}} \approx(100)$ (cf. (4)), there is such a matrix $\widetilde{\mathbf{A}}$, which turns out to be a distance of order $\delta^{2}$ from $S O(3) \mathbf{A}$. However, it also turns out from this calculation that $\widetilde{\mathbf{R}} \neq \mathbf{I}$.

Now consider the dotted line in Figure 2 passing from point $b$ to point $c$. To have a low energy transition layer along this line, we would like to have a deformation gradient that passes from $\widetilde{\mathbf{A}} \approx \widetilde{\mathbf{R}} \mathbf{A}$ to $\mathbf{A}$, and stays near the well $S O(3) \mathbf{A}$, i.e. a deformation gradient approximately of the form $\mathbf{R}(\mathrm{x}) \mathbf{A}$. By general continuum mechanics, there is no deformation precisely of this form, but a bending deformation has such a form on a plane (the neutral surface of the "beam") and slightly differs from this form at points near the plane. This is the proposed deformation in the larger region outside the needles. It turns out by good fortune that the needles themselves also have approximately a bending deformation with gradient near $S O(3) \mathbf{B}$. We believe this is the basic reason for the observed microstructure.

It is not difficult to formalize the description above, that is, to write down a particular deformation that conforms to this description. An example is pictured in Figure 3. To plot this, we have chosen a symmetric shape of the needles in the reference configuration, and we have chosen $\delta$ relatively large $(\delta=0.2)$ so the deformation can be easily seen. The deformed grid is shown in Figure 3. The details of this construction will appear in a forthcoming publication [8]. The fact that the energy is low can be seen qualitatively by following the deformation of the squares of dots. Note that the shear is down, but the bending is up.

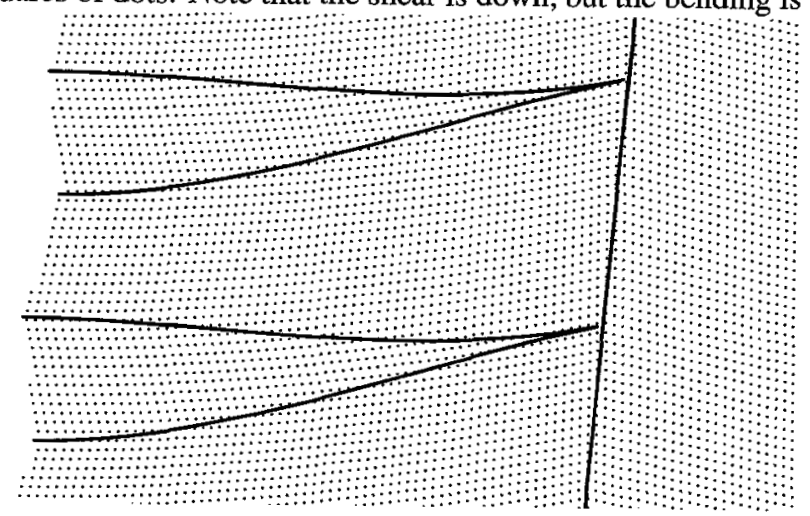

Figure 3. Deformation of the transition layer according to geometrically nonlinear theory.

\section{MODEL OF THE TRANSITION LAYER - GEOMETRICALLY LINEAR THEORY}

For geometrically linear theory, one can make deformations analogous to the one shown in Figure 3, and it is also easier to assess the effects of branching and tip splitting in geometrically linear theory. In fact, the constructions are easier to do, because the analog of $\widetilde{\mathbf{A}}$ described above lies precisely on the well 
$\left(\frac{1}{2}\left(\mathbf{A}+\mathbf{A}^{T}\right)-\mathbf{I}\right)+\mathbf{W}, \mathbf{W}=-\mathbf{W}^{T}$, rather than merely near it. Because of this, one can find a deformation in geometrically linear theory that is exactly pure bending (according to linear elasticity) outside the needles. Furthermore, if one chooses the shape of the needles appropriately, it is possible to have pure bending also within each needle. If we let $\left(u\left(x_{1}, x_{2}, x_{3}\right), v\left(x_{1}, x_{2}, x_{3}\right), w\left(x_{1}, x_{2}, x_{3}\right)\right)$ be the components of the displacement, then a suitable class of deformations have $w=0, u\left(x_{1}, x_{2}+h\right)=u\left(x_{1}, x_{2}\right)+\delta(2 \lambda-1) h$, and are otherwise given by:

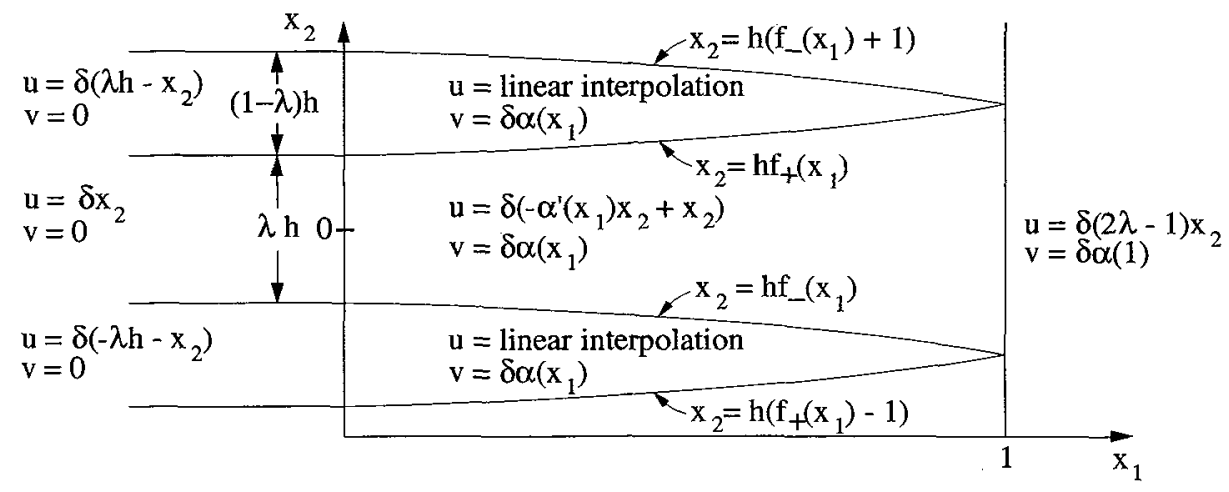

Here, $\alpha(0)=\alpha^{\prime}(0)=0, \alpha^{\prime}(1)=2(1-\lambda)$, and "linear interpolation" refers to the unique function that is linear in $x_{2}$ inside the needles and agrees with the specified displacement at their boundaries. Note that there is no singularity at the tips of the needles. A plot of this displacement (with a suitable choice of the constants and the needle shape: $f_{-}$and $\left.f_{+}\right)$is similar to that shown in Figure 3.

As is well-known, interfacial energy is expected to play an important role in branching of the microstructure under study (see [11]). In analyzing this microstructure we have operated under the principle that the main role of interfacial energy is to set the scale of the microstructure, as found in [11] in a simpler case. A preliminary idea of the role of interfacial energy can be obtained by altering the deformation (7) by locally splitting the tips of the martensite needles (This is easily accomplished because, in all of our deformations, the strain tends to a finite limiting matrix at the needle tip, so locally the deformation of the needle tip looks like a deformation with constant strain sectors). We shall only present the results of this calculation. It is found, as expected based on the fact that it is a "small-scale" calculation, that interfacial energy always favors the absence of splitting, but the energy barrier for splitting decreases as the width/length ratio of the needles increases, i.e., short, fat needles want to split. A quantitative analysis is found in [8]. This agrees at least qualitatively with the observations of [7], where tip-splitting was often observed as the volume fraction $\lambda$ was being changed.

\section{NUMERICAL CALCULATION OF A RELAXED TRANSITION LAYER}

In this section we present a preliminary numerical relaxation of the transition layer, using a bulk energy appropriate to the two compound twinned variants of the $\gamma_{1}^{\prime}$ phase of the $\mathrm{Cu}-14.0 \mathrm{wt} \% \mathrm{Al}-3.9 \mathrm{wt} \% \mathrm{Ni}$ alloy pictured in Figure 1. Because this was taken from a thin plate with normal (100), we have first derived a geometrically nonlinear, plane-stress energy function appropriate to this plate, using the measured moduli of Yasunaga et al. (see [13]) to get the shape of the energy near each well and the measured transformation strain to get the wells themselves. The resulting nonlinear energy for variant one $\left(\mathrm{C}_{11} \geq \mathbf{C}_{22}\right)$ is

$$
\varphi^{1}(\mathbf{C})=7.85 \mathbf{C}_{11}^{2}-11.8 \mathbf{C}_{11} \mathbf{C}_{22}-5.4 \mathbf{C}_{11}-13.3 \mathbf{C}_{22}-8.4 \mathbf{C}_{12}^{2} \mathrm{GPa},
$$

where the components of $\mathbf{C}=\mathbf{F}^{T} \mathbf{F}$ are given in the orthorhombic basis. This energy has wells at $S O(2) \mathbf{U}_{1}$ and $S O(2) \mathrm{U}_{2}$ where $\mathrm{U}_{1}$ and $\mathrm{U}_{2}$ are the matrices,

$$
\mathbf{U}_{1}=\left(\begin{array}{cc}
1.0619 & 0 \\
0 & 1.0230
\end{array}\right), \mathbf{U}_{2}=\left(\begin{array}{cc}
1.0230 & 0 \\
0 & 1.0619
\end{array}\right)
$$


By geometrical linearization (i.e, replace $\mathbf{C}=\mathbf{U}_{1}^{T} \mathbf{U}_{1}+2 \mathbf{U}_{1}^{T} \epsilon \mathbf{U}_{1}+\ldots, c f .[10]$ ) we get the linear energy for variant one

$$
\varphi_{\text {lin }}^{1}=40.0 \epsilon_{11}^{2}-55.7 \epsilon_{11} \epsilon_{22}+55.6 \epsilon_{22}^{2}+39.4 \epsilon_{12}^{2} \quad \mathrm{GPa},
$$

where $\epsilon_{i j}$ is the elastic strains in variant one. The total strain in this variant relative to the reference austenitic state is

$$
\epsilon_{t}=\epsilon+\epsilon_{1}
$$

where $\epsilon_{1}=\mathbf{U}_{1}-\mathbf{I}$ is the transformation strain of variant one. From (10) we can determine the plane-stress moduli for the variant with transformation strains $\mathbf{U}_{1}$. The Young's modulus for tension in the $x_{1}$-direction is $E_{1}=52.1 \mathrm{GPa}$, in the $x_{2}$-direction it is $E_{2}=72.5 \mathrm{GPa}$ and the $x_{1}-x_{2}$ plane shear modulus and Poisson's ratio are $G_{12}=19.7 \mathrm{GPa}$ and $\nu_{12}=-0.50$, respectively.

Equation (11) has the same form as the equations for linear thermo-elasticity with the transformation strain $\epsilon_{1}$ playing the role of the thermal strains. Thus we can solve the linear transition layer problem using any finite element method (FEM) that supports orthotropic materials and thermo-elasticity. I-DEAS by Structural Dynamics Research Corporation (Milford, Ohio) is one such FEM package that solves these types of problems. Using the orthotropic elastic constants identified above and setting in-plane thermal expansion coefficients in the $x_{1}$ and $x_{2}$ directions to be $\alpha_{1}=0.0619$ and $\alpha_{2}=0.0230$ correctly models the linearized problem. The temperature difference for the problem was set to 1.0 .

The constants identified above are for the variant with transformation strain $\epsilon_{1}$. The second variant (with transformation strain $\epsilon_{2}=\mathbf{U}_{2}-\mathbf{I}$ ) is symmetry related to the first variant by a rotation of 90 degrees about the $x_{3}$ axis. Thus a single material is used in the FEM model and the two variants have material orientations that differ by 90 degrees. In Figure 2 the twin planes are horizontal and vertical. To match this orientation the material axes in the FEM model will be taken to be \pm 45 degrees from the horizontal direction for the two variants. One final adjustment was made in order to make application of periodic boundary conditions in the (010) direction of Figure 2 simple. The dilatational component of the transformation strain is same in both variants, thus this component results in a uniform in-plane expansion of the entire microstructure and no stresses being developed. Removing the dilatational component from the transformation strains results in a pure shear deformation. This procedure gives $\alpha_{1}=0.01945$ and $\alpha_{2}=-0.01945$ as the thermal expansion coefficients in the FEM model.

In order to obtain a suitable starting geometry, experimental data similar to that shown in Figure 1 was used to obtain the shape of the deformed needles. Using the theory presented above which assumes that the bent shape is due to elastic deformations, the bending of the needle was removed by subtracting a pure bending deformation. This resulted in an almost perfectly symmetrical reference shape for the needle. This shape can be approximated very well by the following function

$$
f_{+}\left(x_{1}\right)=\frac{w}{2}\left(1-\mathrm{e}^{-\beta x_{1}}\right),
$$

where $w$ is the far field width of the needle and $\beta$ is the decay rate. The fit of the observed needle gave $w=1.57$ and $\beta=0.172$, these values are used in all the calculations below, where the length of the needles is 20 in the arbitrary units of the FEM model.

The vertical interface in Figure $1\left(x_{1}=1\right.$ in equation 7$)$ is noticeably wavy with the same period as the needles. The shape of this interface is primarily due to its shape in the reference configuration. To determine if a given shape for an interface is the equilibrium shape, we must determine not only a solution to the equations of linear thermo-elasticity (equilibrium and compatibility) but we must also allow the shape of the interface in the reference configuration to vary and pick the interface shape that results in the lowest free energy in the solution. By considering the motion of an interface in the reference configuration (note that interfaces between variants are not material) we can find the driving traction $F_{d}$ on an interface [1],

$$
F_{d}=\llbracket \varphi_{\text {lin }} \rrbracket-\operatorname{tr}(\sigma \llbracket \epsilon \rrbracket)
$$

where $\llbracket f \rrbracket=f(x+)-f(x-)$ is the jump in a quantity across an interface and tr is the trace of a $3 \times 3$ matrix. A positive value of the driving traction means that more strain energy would be released than the work needed to transform the material as the interface moves toward the positive side of the interface. Thus it is energetically favorable for the interface to move in this direction. We will assume that the velocity of 
the interface is proportional to the driving traction. The proportionality constant will be chosen to give a reasonable amount of change in the interface shape.

Figure 4 shows the shear strain $\epsilon_{12}$ in variant $A$ as gray scales on the deformed geometry. This planestress calculation was performed using the material constants given above and for the needle shape given in (12). The boundary conditions are symmetric on the top and bottom edges and free on the sides. The needles shown have a volume fraction of $1 / 2$. The bending in the needles, which are symmetric in the reference configuration, is clearly visible because the deformations have been multiplied by a factor of 2 for this figure. Without magnification the amount of bending is very similar to the amount of bending observed in needles with a volume fraction of $1 / 2$ in $\mathrm{Cu}-\mathrm{Al}-\mathrm{Ni}$. This solution is the second iteration of changing the shape of the vertical interface. Figure 5 shows the driving traction along the vertical interface. The solid lines are the values of the driving traction when the interface is straight in the reference configuration. By changing the shape of the interface in proportion to the the driving traction, the driving traction was reduced to the amount shown by the dashed curve in Figure 5.

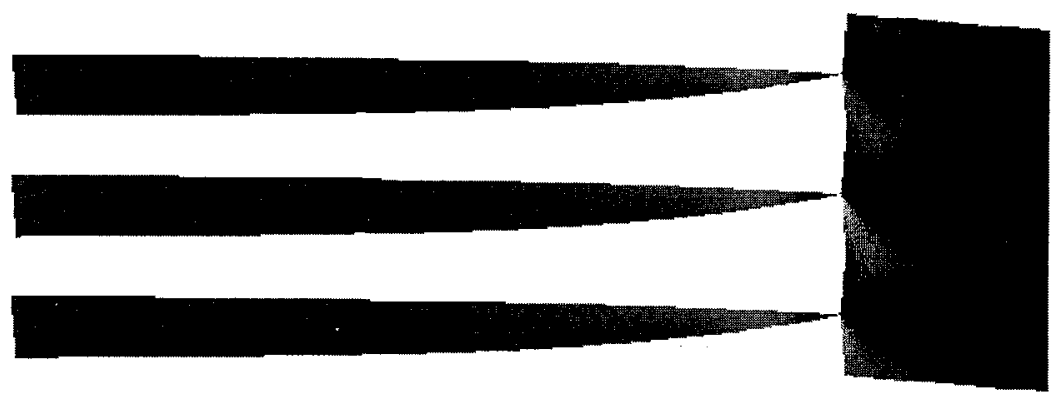

Figure 4. The shear strain $\epsilon_{12}$, black $=-.02$, white $=-.0185$, on the deformed geometry.

The overall elastic energy in the solution dropped by 4.3 percent due to this small change in the shape of the vertical interface. Calculations of the driving traction on the needle interfaces show that they are not exactly in equilibrium but are reasonably close. This calculation also shows that the needle will remain symmetric in the reference configuration because the driving traction is symmetric. This suggests that the bending of the needles is due to elastic deformations, whereas the waviness of the vertical interface is due to its shape in the reference state. For more details on the methods used to calculate the updated interface shapes and the results of a detailed study of these types of equilibrium microstructures see [8].

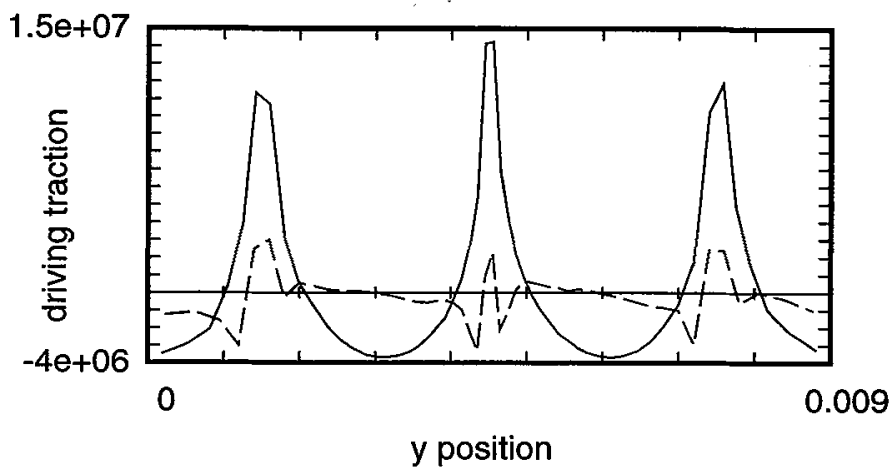

Figure 5. The driving traction on the vertical interface in Figure 4. Each peak is at the tip of a needle.

\section{Acknowledgment}

The authors thank ONR (N00014-93-1-0240, NOOO14-91-J-4034), AFOSR (AFOSR-91-0301), NSF (CMS95-03633, DMS-9111572-02, DMS-9402763, DMS-9404376, CM5-9257945-03) and ARO (DAAH04-95-1-0100) for supporting this work. 


\section{References}

[1] Abeyaratne R., J. of Elasticity 13 (1983), pp. 175-184.

[2] Abeyaratne R., Chu C. and James R. D., submitted to Phil Mag.

[3] Ball J. M. and James R. D., Archive for Rational Mechanics and Analysis 100 (1987), pp. 13-52.

[4] Ball J. M. and James R. D., Phil. Trans. Royal Soc. London A 338 (1992), pp. 389-450.

[5] Ball J. M. and James R. D., International Conference on Martensitic Transformations, July 20-24, 1992, C. M. Wayman and J. Perkins Eds. (Monterey Institute for Advanced Studies, 1993) pp. 65-76.

[6] Bhattacharya K., Continuum Mech. Thermody. 5 (1993), pp. 205-242.

[7] Chu C., Ph.D. Thesis, University of Minnesota (1993).

[8] Chu C., James R. D., Kohn R. V. and Shield T. W., in preparation.

[9] Khachaturyan A. G., Sov. Phys. Solid State 8 (1967), pp. 2163-2168.

[10] Kohn R. V., Cont. Mech. Thermody. 3 (1991), pp. 981-1000.

[11] Kohn R. V. and Müller S., Phil. Mag. A 66 (1992), pp. 697-715.

[12] Roitburd A. L., Solid State Physics 33 (1978), pp. 317-390.

[13] Yasunaga M., Funatsu Y., Kojima S., Otsuka K. and Suzuki T., J. de Physique 43 (1982), pp. 603-608. 